\title{
The early response to a novel coronavirus in the Middle East
}

A. Mounts, ${ }^{7}$ S. De La Rocque, ${ }^{2}$ J. Fitzner, ${ }^{7}$ E. Garcia, ${ }^{3}$ H.L. Thomas, ${ }^{4}$ D. Brown, ${ }^{5}$ H. Schuster, ${ }^{6}$ K. Vandemaele, ${ }^{7}$ H. Esmat, ${ }^{7}$ S. Eremin ${ }^{8}$ and A. Mafi ${ }^{9}$

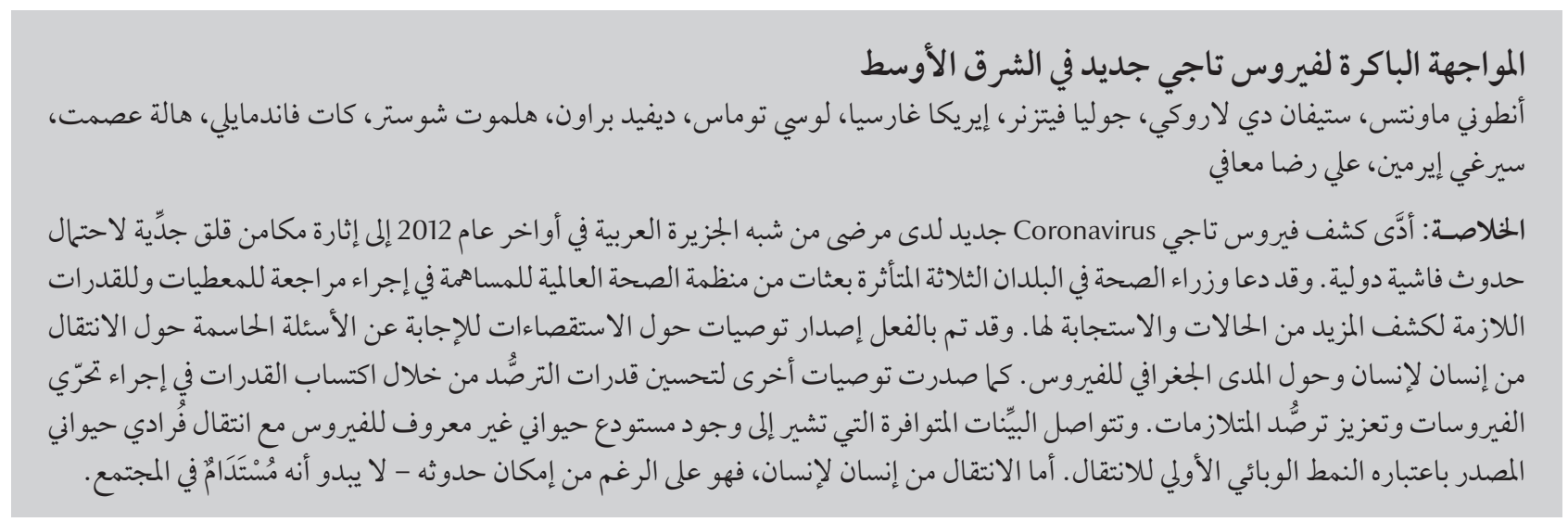

ABSTRACT The detection of a novel coronavirus in patients from the Arabian Peninsula in late 2012 raised serious concerns of a possible international outbreak. Ministries of health of the three affected countries invited missions from the World Health Organization to participate in a review of data and capacity to detect and respond to further cases. Recommendations were made for investigations to answer critical questions about human-tohuman transmission and the geographic extent of the virus. Additional recommendations were made to improve surveillance capacity by acquiring the capacity to test for the virus and enhance syndromic surveillance. Available evidence continues to suggest an unknown animal reservoir for the virus with sporadic zoonotic transmission as the primary epidemiological pattern of transmission. Human-to-human transmission, while it can occur, does not appear to be sustained in the community.

\section{Riposte rapide au nouveau coronavirus au Moyen-Orient}

RÉSUMÉ La détection d'un nouveau coronavirus chez des patients de la Péninsule arabique à la fin de l'année 2012 a soulevé de graves inquiétudes concernant une possible flambée internationale. Les ministères de la Santé des trois pays affectés ont invité des missions de l'Organisation mondiale de la Santé à participer à une revue des données et à un examen des capacités pour dépister les cas à venir et y riposter. Des recommandations ont été formulées pour les recherches visant à répondre aux questions critiques sur la transmission interhumaine et la propagation géographique du virus. Des recommandations supplémentaires ont été effectuées afin d'améliorer les capacités de surveillance en développant la capacité de détection du virus et en renforçant la surveillance syndromique. Les données dont nous disposons semblent toujours indiquer un réservoir animal viral inconnu avec un mode de transmission zoonotique sporadique comme principal modèle épidémiologique de transmission. La transmission interhumaine, si elle est possible, ne semble pas soutenue au sein de la communauté.

${ }^{7}$ Global Influenza Programme; ${ }^{2}$ IHR Monitoring, Procedure and Information; ${ }^{3}$ Alert and Response Operations; ${ }^{8}$ Infection Prevention and Control in Health Care, World Health Organization, Geneva, Switzerland (Correspondence to A. Mounts: mountsa@who.int).

${ }^{4}$ Field Epidemiology Training Programme; ${ }^{5}$ Virus Reference Department, Public Health England, London, United Kingdom.

${ }^{6}$ Public Health England, University Hospital Southampton NHS Foundation Trust, Southampton, United Kingdom.

${ }^{7}$ Public Health Laboratory Unit, World Health Organization Regional Office for the Eastern Mediterranean, Cairo, Egypt.

${ }^{9}$ Pandemic and Epidemic Disease, Division of Communicable Disease Control, World Health Organization Regional Office for the Eastern

Mediterranean, Cairo, Egypt. 


\section{Background}

The detection of a novel coronavirus in two patients from the Arabian Peninsula in late 2012 captured the attention of the global public health community for a number of reasons. The virus was of the same family that was responsible for a large multinational outbreak of severe acute respiratory syndrome (SARS) in 2003 and while the novel virus was not closely related genetically, the clinical syndrome associated with the early confirmed cases was severe $[1,2]$. In addition, the first two reported cases had a history of having been in the area around Mecca and Medina in the weeks before their illnesses and the second case occurred close in time to the Hajj. The subsequent retrospective confirmation of an outbreak among health care workers (HCW) in Jordan with two confirmed and a number of probable cases raised concerns that the virus could be transmissible between humans. The appearance of the virus in three widely separated areas over at least a six-month period further demonstrated the virus persistence and wide distribution. These concerns prompted the ministries of health of all three countries (Saudi Arabia, Qatar and Jordan) to invite teams of international experts to review their data and capacity to detect and respond to a serious outbreak of a novel pathogen.

\section{Primary questions}

At the outset, the working hypothesis regarding the emergence of the novel coronavirus was that it had a zoonotic origin with animal host(s) and sporadic spill-over into humans. However, the evidence for this was entirely circumstantial. If this was not a zoonosis, the sporadic appearance of severe human cases with long periods of time between them and the wide area over which the virus was apparently distributed would have required unrecognized ongoing transmission in the human population to explain the pattern of occurrence. Furthermore, early comparisons with other known coronaviruses suggested a similarity to viruses previously described in bats, although this may only reflect the paucity of coronavirus sequences available in public databases for comparison [3]. Finally, it has been demonstrated that the virus could be easily grown in a number of widely used cell lines [4], and therefore would have likely been detected earlier through routine diagnostic testing if circulating widely in human populations over a long period of time. However, the use of cell culture as a standard diagnostic tool has decreased markedly in the past decade in favour of polymerase chain reaction (PCR), which is much less likely to detect novel pathogens.

The appearance of a novel pathogen can have serious public health and economic impacts but the immediate concern is to assess its potential international implications as described in the International Health Regulations, 2005 [5]. As such, the primary questions to be addressed early in the course of an event include: i) does the pathogen transmit from human to human, if not, ii) what is its geographic distribution and iii) what are the risk factors for acquiring infection. For a pathogen to have truly pandemic potential it would need to be easily transmissible from human to human. However, even if nontransmissible among humans, a zoonosis that is widespread in an animal population could cause widespread disease among humans, particular if contact between human and animal is frequent or transmission is easy. Answering these critical questions requires thorough field investigation to determine the exposure(s) that result in infection, investigate whether human-to-human transmission has occurred, search for unrecognized cases and detect signals that may represent previously unrecognized circulation of the novel pathogen in the community (Table 1).

\section{Initial missions}

The initial assessment by the World Health Organization (WHO) was aimed primarily at reviewing the data already collected during investigations by the respective ministries of health in Saudi Arabia, Qatar and Jordan to evaluate the possibility that this event constituted a Public Health Emergency of International Concern (PHEIC) as defined in the IHR (2005). In addition, the WHO missions sought to support the ministries by assisting in a review of their capacity to detect and respond to potential further emergence of the novel pathogen. The information reviewed included:

- The histories given by the cases of exposures to animals, potential environmental sources, and other sick humans in the days before infection, including a history of travel outside of the area where illness first occurred.

- Data from investigations of close contacts of the known cases, including members of the household, close social contacts and HCWs exposed in the hospital.

- Historical trends in data from hospital and intensive care unit admissions for severe respiratory infections, mortality from pneumonia and the occurrence of severe respiratory disease reported to sentinel surveillance systems.

- Hospital infection control policies and procedures.

- Respiratory disease surveillance system procedures, policies and capacity, including the capacity to incorporate testing for novel viruses in the laboratory.

\section{Answering the critical questions}

Several recommendations were made by the missions to specifically address the critical questions (Table 1). 


\begin{tabular}{|c|c|}
\hline Question & Investigation \\
\hline Does human-to-human transmission occur? & $\begin{array}{l}\text { - Survey of exposed health care workers with serological and } \\
\text { virological testing } \\
\text { - Contact tracing of exposed family and social contacts } \\
\text { supported by serological testing }\end{array}$ \\
\hline What exposures result in infection? & $\begin{array}{l}\text { - Interview of cases or proxies; case-control study of } \\
\text { exposures }\end{array}$ \\
\hline What is the source of the novel pathogen? & $\begin{array}{l}\text { - Virological and serological testing of animals, food stuffs; } \\
\text { environmental sampling } \\
\text { - Seroepidemiological surveys of specific human risk groups } \\
\text { - Testing of stored animal clinical specimens }\end{array}$ \\
\hline Is the pathogen new? When did it first appear? & $\begin{array}{l}\text { - Review of recent surveillance data, admission records and } \\
\text { vital statistics data for the relevant clinical syndrome } \\
\text { - Retrospective testing of stored clinical specimens from } \\
\text { studies, surveillance or health care facility archives }\end{array}$ \\
\hline
\end{tabular}

\section{Human-to-human transmission}

The first clue to both the source of infection and the presence of humanto-human transmission will often occur in the initial interview with the patient and family. Patients or their proxies should be carefully questioned using memory prompts such as calendars, menus, travel records, etc. about recent travel, exposure to sick individuals, exposure to animals, activities, hobbies, and consumption of unprocessed foods and beverages. Questions should initially be open-ended and broad in scope but may become more specific as hypotheses are generated about possible sources of infection. Ultimately, exposures of interest identified during the interview process can be tested for significance through carefully designed case-control studies comparing rates of exposure in the cases to rates in similar randomly selected individuals from the community. Members of the household and others with close physical contact should be asked about symptoms occurring around the same time as the known case. If symptoms are reported, virological testing can be used to diagnose infection, if the time frame is appropriate; if the window of opportunity for virological testing has passed, serological testing will be required.

There are several challenges to determining whether cases or clusters of cases have acquired infection through human-to-human transmission. Primary among these is the fact that a clear history of exposure to potential sources of virus may not be remembered, cases are often incapacitated or dead, and in household clusters, the majority of the members of a household will likely share common environmental exposures. Investigation of the first reported case was complicated by the fact that he had died many months prior to discovery of the virus that caused his illness. The second case, however, offered a unique opportunity to tease out potential sources of infection and help clarify the incubation period. His history illustrates the utility of household investigations. The patient had travelled with select members of his household to Mecca and Medina, the same location where the first case may have acquired infection, two to four weeks prior to illness onset. He then returned home to Doha but also made a visit with other members of his household to a family farm outside the city. Different members of his household had three different types of exposure: exposure to the same environment in Mecca/ Medina, exposure to the household environment in Doha but not Mecca and not the farm, and exposure to the farm but not Mecca / Medina or Doha (farm workers).
Because of the challenges in disentangling potential sources of infection in household investigations, HCWs caring for patients with the novel infection may provide the best first evidence of human-to-human transmission. Careful exposure histories using standard questionnaires to document duration and intensity of exposure and the use of personal protective equipment are important. If carried out prospectively, PCR testing can be used to test symptomatic workers exposed to the patient. However, the more likely scenario will involve retrospective investigations of workers exposed before recognition of the novel agent and subsequent implementation of enhanced infection control. In this situation, serological assays are used to confirm infection. As acute and convalescent sera may not be possible and diagnosis may rely on single sera, the use of control groups chosen from a similar group of unexposed workers will enable investigators to control for rates of background seropositivity and exposures occurring external to the health care environment.

\section{Is the agent a novel pathogen?}

While a newly recognized agent is presumed to be a novel pathogen, nonspecific clinical presentation and the absence of pre-existing laboratory tests require a careful search for 
evidence of previous circulation to confirm that it is indeed novel. In the case of $\mathrm{nCoV}$, the most commonly recognized clinical presentation is severe respiratory disease. However, even in prospective studies, the etiology of most community acquired pneumonias are not proven and patients are very often treated empirically $[6,7]$. Consequently, single, sporadic infections with novel respiratory pathogens may be undetectable if not investigated as thoroughly as the first diagnosed case of $\mathrm{nCoV}$. However, the appearance of a novel transmissible agent may cause a demonstrable increase in background rates of respiratory disease. A review of temporal trends in hospital admissions, deaths in the community and cases reported to surveillance systems, such as the sentinel system for Severe Acute Respiratory Infection (SARI), can provide clues as to the timing of the first appearance of novel respiratory pathogens. In addition, stored specimens previously collected from similar cases either as part of specific studies or ongoing surveillance programmes can be tested retrospectively for the presence of the virus. Such strategies have been used to push back the time of first appearance of human immunodeficiency virus (HIV) in Africa. [8]. Retrospective testing of specimens collected in an earlier outbreak of respiratory disease in Jordan demonstrated that the $\mathrm{nCoV}$ had been present longer than initially thought. However, review of pneumonia admission records, admissions to intensive care for respiratory disease, vital statistics data and data from the SARI surveillance system all indicated that background rates of respiratory disease in the three affected countries had been stable in recent years and had not increased notably since the first appearance of the virus. This would indicate that either the agent has not been previously circulating in the community or that the number of cases has been low enough or mild enough that they are lost in the "background noise" of disease caused by a wide variety of pathogens. The absence of a post-introduction surge in cases does provide reassurance that the virus was not transmitting with the same degree of frequency as seen in SARS. Retrospective testing of stored SARI specimens indicated that the virus had not been widely circulating prior to the recognition of the first cases [Jordan Ministry of Health unpublished data]

\section{Source of the virus}

Several recent zoonotic outbreaks have indicated how complicated transmission from a zoonotic source to humans can be. In SARS, the endemic source of the virus was ultimately discovered to be horseshoe bats. However, palm civets were initially implicated as the source of transmission to humans and humanbat exposure likely did not play a direct role in the outbreak $[9,10]$. Likewise, the Nipah virus has displayed circuitous transmission routes from bat to human. In Malaysia, swine acted at the intermediary vector and amplifier of infection [11]. In Bangladesh, raw palm sap contaminated with bat guano has served as a vector [12]. In the $\mathrm{nCoV}$ event, both of the first two reported cases had a history of having visited farms with a variety of animals in the two weeks prior to illness. However, no direct exposure to animals was reported and no illness was reported in the animals on the farms. In addition, many thousands of animals are imported into the region every year both for consumption and for ritual sacrifice, and an animal reservoir need not exist in the country where the cases were reported in order to result in exposure.

Finding the animal source of a zoonotic virus can be even more complicated when associated with wildlife and a clear route of transmission can be difficult to find as demonstrated by the example of Nipah virus in Bangladesh noted above. However, capture and testing of animals from the environment of cases is often the first step in identifying the reservoir of a novel agent. In addition, retesting of stored diagnostic specimens from animal health laboratories or from importation screening can provide evidence of both the species of animal and the geographic origin of the virus if external to the country where the cases have occurred, once an appropriate test is developed.

\section{Secondary investigations}

Even before the public health importance of a novel pathogen is completely understood, there are a number of critical secondary questions that need to be addressed in order to be adequately prepared to respond in case of accelerating transmission (Table 2). These are questions that will inform control measures and include the incubation period, mode of transmission, degree of transmissibility, the spectrum of disease severity, and natural history of infection. The data related to these characteristics of the novel pathogen will determine the need for and duration of isolation and quarantine, inform case definitions for case finding and other containment strategies, such as social distancing, if they are needed.

\section{Recommendations for system enhancements}

There are also several areas in which enhancements are likely to be needed to existing systems in order to monitor for future cases and enable appropriate management (Table 3).

\section{Surveillance}

The primary goal of surveillance enhancements in the context of a novel pathogen should be to monitor for the occurrence of human-to-human or accelerating transmission. Two situations that can be useful indicators are clusters of cases with a compatible clinical presentation and the occurrence of disease in HCWs. Tight clusters of severe 


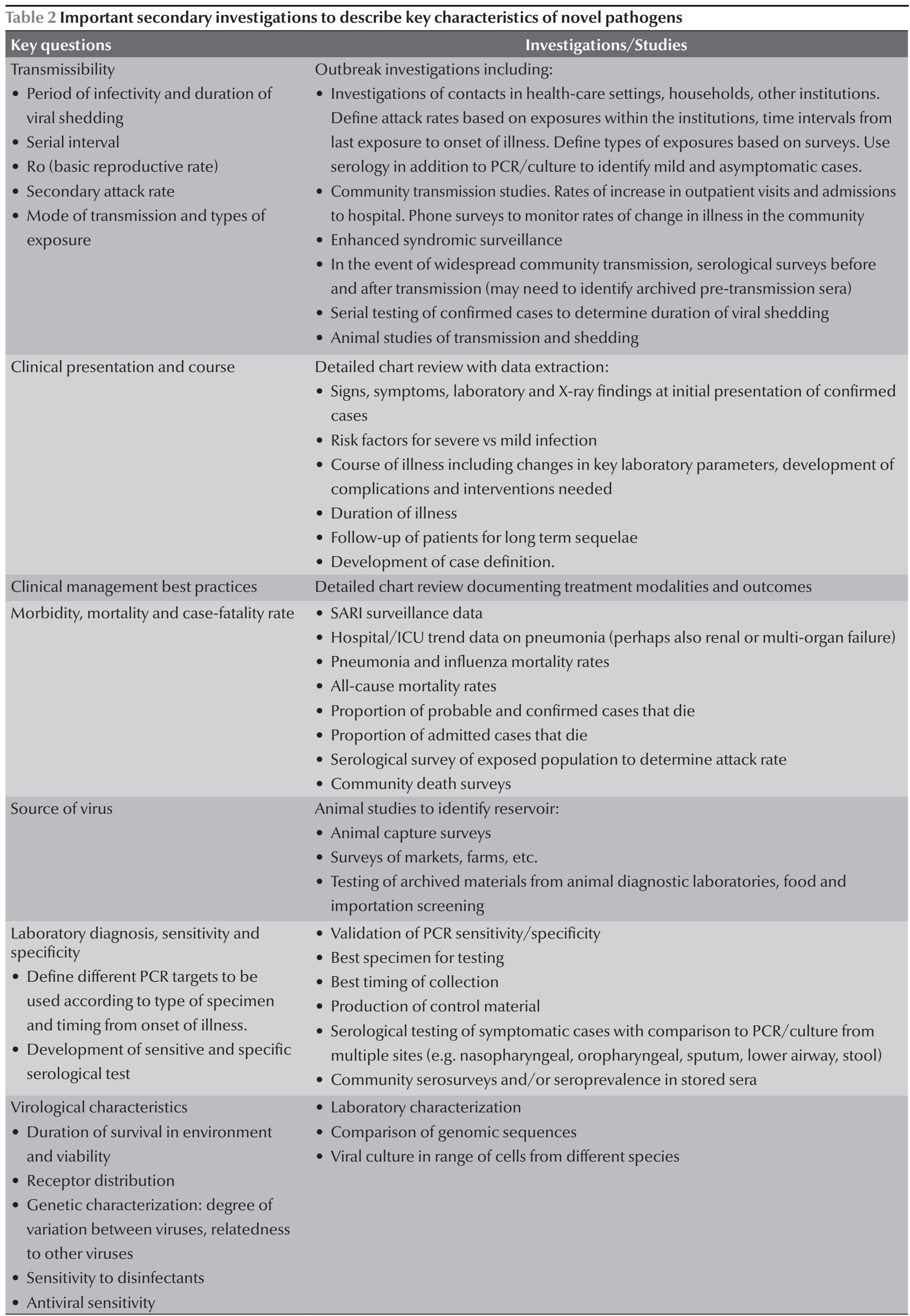

$I C U=$ intensive care unit; $S A R I=$ Severe Acute Respiratory Infection; $P C R=$ polymerase chain reaction 


\begin{tabular}{ll}
\hline Table 3 Recommendations for enhancing detection capacity and preparedness for response to novel pathogens \\
\hline Domain
\end{tabular}

respiratory disease occurring in small groups of individuals such as a family or classroom are an unusual event outside of chronic care facilities and generally represent an event of public health importance [13]. Pneumonia related to seasonal influenza, pneumococci, haemophilus influenza infections and other commonly recognized pathogens typically does not occur in multiple members of the same family, classroom or work place in a short period of time. As such, clusters always deserve investigation. Indeed, the occurrence of a family cluster was the first indication of the appearance of influenza $\mathrm{A}(\mathrm{H} 7 \mathrm{~N} 90)$ in China in April 2013. HCWs likely acquire pneumonias at a similar rate as other adults of similar age and health status. However, due to the fact that they may have a higher risk of exposure to human sources of infection and are not from the same home environment as the cases for whom they care, they may provide a useful marker of humanto-human transmission for novel pathogens.

The presence of a pre-existing surveillance system for SARI greatly facilitates the establishment of a system to monitor for widespread community transmission and real-time detection of a sudden surge in cases. These systems are usually established for influenza monitoring and use molecular techniques to test for influenza viruses as part of the system [14]. They provide baseline data on the occurrence of respiratory disease in the community and can be adapted for monitoring $\mathrm{nCoV}$ by the incorporation of specific testing into the operating procedures. In the case of $\mathrm{nCoV}$, limited evidence from early cases indicated that the virus might be more easily detected in lower respiratory tract specimens necessitating a slight adjustment of specimen collection practice in the existing system [15]. The addition of testing for $\mathrm{nCoV}$ as a routine diagnostic tool for community acquired pneumonias used at the discretion of the treating clinician in areas where the virus is thought to be present can also provide additional monitoring.

Enhancing laboratory capacity in the age of molecular techniques such as PCR is often a matter of incorporation of new primers into existing testing panels. However two important considerations are required in the context of a novel pathogen. The first is biosecurity in the extraction of RNA. With a novel agent, especially one known to cause severe and fatal disease, at least a BSL2 facility including use of a microbiological safety cabinet (class 1,2 , or 3 ) is required for the handling of potentially infectious materials. While this is beyond the capacity of most routine virological diagnostic laboratories, the facility may be available in laboratories handling tuberculosis specimens. In addition, the widespread use of multiplex PCR platforms may complicate the introduction of new primers for novel agents. The interplay between different primers and reagents in a multiplex PCR platform requires a great deal of testing and validation. The primers for novel agents will initially have to be tested in a separate PCR to determine optimal cycling conditions before being incorporated in the multiplex PCR. Finally, the interpretation of PCR results can be problematic as their sensitivity and relationship to actual infection is generally unknown early in the course of an event with a novel pathogen. Without actual culture and the support of serology to document seroconversion, it may prove difficult to clearly know that infection has in fact occurred with a positive PCR.

\section{Infection control}

Health care facilities played an important role in the transmission of SARS in the 2003 outbreak and the implementation of rigorous infection control practices was critical to breaking chains of transmission. The successful prevention of amplification of $\mathrm{CoV}$ infections associated with health care facilities will depend on the full implementation of all core components for infection prevention and control (IPC) programmes 16]. Most nosocomial transmissions occur in the absence of basic IPC precautions and before a specific infection is suspected or confirmed, hence the routine application of standard IPC precautions and additional measures to prevent spread of acute respiratory infections (ARI) when caring for symptomatic patients is essential to reduce the spread of any ARI in health-care 
settings. These as well as the additional precautions to be applied when caring for patients with probable or confirmed infection with $\mathrm{nCoV}$ are discussed in detail in the accompanying article in this issue of EMHJ by Seto et al. on infection prevention and control measures [17].

\section{Conclusions}

Early investigations into the appearance of a novel coronavirus in the Middle East indicated that sustained community transmission was not occurring. Review of the available data suggested that while nosocomial human-to-human transmission might have occurred in Jordan in April 2012, transmission did not appear to occur frequently or easily. Enhancements to surveillance including acquiring the appropriate primers for diagnosing infection with the novel agent have enabled the affected countries to detect further cases which have not markedly changed the initial assessment. However, further vigilance is needed along with expanded testing to determine the full geographic extent of the virus. The source of the virus remains a mystery that urgently needs to be solved.

The appearance of a novel pathogen carries many inherent risks over and above those to global public health that create a reluctance by national authorities to announce its appearance. The economic impact can be substantial as demonstrated in Mexico when the novel influenza $\mathrm{A}(\mathrm{H} 1 \mathrm{~N} 1)$ virus first appeared [18]. As such, the risk to global public health from a novel pathogen with unknown potential for international spread must be balanced with the risk to local economies from an exaggerated and unnecessary response from a concerned public. The most effective way to mitigate the negative impact of an emerging novel pathogen is through rapid investigation and response with the support of international partners, as needed. The International Health Regulations ratified by WHO Member States in 2005 established a mechanism for the confidential sharing of information between WHO and other Member States early in an event before its significance is even fully evaluated. This greatly reduces disincentives to information sharing and facilitates global cooperation. The SARS experience of 2003 taught many lessons about how to respond to global crises. The elements that made the response successful included the open sharing of information internationally, the rapid deployment of teams to support and supplement manpower in affected countries, and the global coordination of research activities and the sharing of results before their publication so that they could inform response efforts. Answering the critical questions around this novel pathogen will require similar efforts

\section{References}

1. Corman $\mathrm{V}$ et al. Detection of a novel human coronavirus by real-time reverse-transcription polymerase chain reaction. Eurosurveillance, 2012, 17(39):pii 20285.

2. Zaki A et al. Isolation of a novel coronavirus from a man with pneumonia in Saudi Arabia. New England Journal of Medicine, 2012, 367(19):1814-1820.

3. Public Health England. Health Protection Agency. Genetic sequence information for scientists about the novel coronavirus 2012 (http://www.hpa.org.uk/Topics/InfectiousDiseases/ InfectionsAZ/NovelCoronavirus2012/, accessed 6 May 2013).

4. Müller MA et al. Human coronavirus EMC does not require the SARS-coronavirus receptor and maintains broad replicative capability in mammalian cell lines. mBio, 2012, 3(6):1-5.

5. International Health Regulations, 2005, 2nd ed. Geneva, World Health Organization, 2005:43

6. Mandell LA et al. Infectious Diseases Society of America/ American Thoracic Society Consensus Guidelines on the Management of Community-Acquired Pneumonia in Adults. Clinical Infectious Diseases, 2007, 44(Suppl. 2):S27-S72.

7. Bartlett JG et al. Practice guidelines for the management of community-acquired pneumonia in adults. Clinical Infectious Diseases, 2000, 31:347-382.

8. Zhu T et al. An African HIV-1 sequence from 1959 and implications for the origin of the epidemic. Nature, 1998, 391:594-597.

9. Guan $\mathrm{Y}$ et al. Isolation and characterization of viruses related to the SARS coronavirus from animals in southern China. Science, 2003, 302(5643): 276-278.
10. Li W et al. Bats are natural reservoirs of SARS-like coronaviruses. Science, 2005, 310(5748):676-679.

11. Parashar UD et al. Case-control study of risk factors for human infection with a new zoonotic paramyxovirus, Nipah virus, during a 1998-1999 outbreak of severe encephalitis in Malaysia. Journal of Infectious Diseases, 2000, 181:1755-1759.

12. Luby SP et al. Foodborne transmission of Nipah virus, Bangladesh. Emerging Infectious Diseases, 2006, 12(12):1888-1894.

13. Puro VGE. Clustered cases of pneumonia among healthcare workers over a 1-year period in three Italian hospitals: applying the WHO SARS alert. Infection, 2006, 34(4):219-221.

14. Interim epidemiological surveillance standards for influenza. Geneva, World Health Organization, 2012.

15. Bermingham A et al. Severe respiratory illness caused by a novel coronavirus, in a patient transferred to the United Kingdom from the Middle East, September 2012. Eurosurveillance, 2012, 17(40):pii 20290.

16. Core components of infection prevention and control programmes in health care. Aide-memoire. Geneva, World Health Organization, 2011 (Aide-memoire) (http://www.who.int/csr/ resources/publications/AM_CoreCom_IPC.pdf, accessed 6 May 2013).

17. Seto $\mathrm{WH}$ et al. Infection prevention and control measures for acute respiratory infections in healthcare settings: an update. Eastern Mediterranean Health Journal, 2013, 19(Suppl.):S39-S47

18. Flu and the Mexican economy; a painful tune. The Economist, 1 May 2009. 